\title{
Branches of solutions for an asymptotically linear elliptic problem on $\mathbb{R}^{N}$
}

\author{
Youyan Wan \\ Department of Mathematics \\ Jianghan University \\ Wuhan, Hubei, China \\ youyanwan@yahoo.com.cn
}

\begin{abstract}
We consider the following nonlinear schrödinger equation

$-\Delta u+\lambda V(x) u=f(x, u)$ with $u \in H^{1}\left(\mathbb{R}^{N}\right)$ and $u \not \equiv 0,(*)$
\end{abstract}

where $\lambda>0$ and $f(x, s)$ is asymptotically linear withrespect to $s$ at origin and infinity. The potential $V(x)$ satisfies $V(x) \geq V_{0}>0$ for all $x \in \mathbb{R}^{N}$ and $\lim _{|x| \rightarrow+\infty} V(x)=V(\infty) \in(0,+\infty)$. We provethat problem $(*)$ has two connected sets of positive and negative solutions in $\mathbb{R} \times W^{2, p}\left(\mathbb{R}^{N}\right)$ for somep $\in[2,+\infty) \cap\left(\frac{N}{2},+\infty\right)$.

Keywords-Bifurcation, asymptotically linear, Fredholm operator of index zero.

\section{INTRODUCTION}

In this paper, we consider the following nonlinear Schrödingerequation

$$
\left\{\begin{array}{c}
-\Delta u+\lambda V(x) u=f(x, u), \\
u \in H^{1}\left(\mathbb{R}^{N}\right), \quad N \geq 3,
\end{array}\right.
$$

where $\lambda>0$ and the functions $\mathrm{V}$ and $f$ satisfy the following assumptions:

$\left(V_{1}\right) V(x) \in C\left(R^{N}, R\right)$ and there exists $V_{0}>0$ such that $V(x) \geq V_{0}>0$ for all $x \in \mathbb{R}^{N}$;

$\left(V_{2}\right) \lim _{|x| \rightarrow+\infty} V(x)=V(\infty) \in(0,+\infty)$ and eas $\left\{x \in \mathbb{R}^{N}: V(x)<\right.$ $V(\infty)\}>0$;

$\left(F_{1}\right) f(x, s) \in C\left(\mathbb{R}^{N} \times \mathbb{R}, \mathbb{R}\right)$ and $(x, \cdot) \in C^{1}(\mathbb{R}, \mathbb{R}) ;$

$\left(F_{2}\right)$ there exist two functions $h, g \in L^{\infty}\left(\mathbb{R}^{N}\right)$ such that $\lim _{s \rightarrow 0} \frac{f(x, s)}{s}=h(x)$ and $\lim _{|s| \rightarrow+\infty} \frac{f(x, s)}{s}=g(x)$ uniformly in $x \in \mathbb{R}^{N}$, where $h$ and $g$ satisfy

(G) Setting $\quad \Gamma=\inf f \int_{\mathbb{R}^{N}}\left(|\nabla u|^{2}+V(x) u^{2}\right) d x: u \in$ $H^{1}\left(\mathbb{R}^{N}\right)$ and $\left.\int_{\mathbb{R}^{N}} u^{2} d x=1\right\}$, there exists $\alpha \in(\Gamma, V(\infty))$ such that $_{|x| \rightarrow+\infty} g(x)=\operatorname{limf}_{x \in \mathbb{R}^{N}} g(x)=\alpha$;

(H) $|h|_{\infty}<\frac{\alpha V_{0}}{V(\infty)}$.

$\left(F_{3}\right) h(x) \leq \frac{f(x, s)}{s} \leq g(x)$ for $\operatorname{all}(x, s) \in \mathbb{R}^{N} \times \mathbb{R} \backslash\{0\}$.

The existence of solutions of problem (1.1) has beeninvestigated extensively. For problem (1.1) with potentialwell and various conditions on $f(x, u) \not \equiv f(u)$, several authorshave obtained the existence of solutions for large $\lambda$ byvariational methods, for example, [1], [2], [3], [5]. And other authors have got the existence of solutions for $\lambda$ is not necessarily large by concentration compactnessargument and mountain pass geometry, for instance, [7], [8]. Stuart and Zhou [10] havestudied how the positive and negative solutions of problem(1.1)depend on $\lambda$ by topological methods.

Inspired by the results we mentioned above, the main object of thisarticle is to investigate the relation between the positive andnegative solutions of problem (1.1)and the parameter $\lambda$, where the potential need not be well potential and $f(x, u)$ is asymptotically linear with respect to $u$ at origin andinfinity.

For this purpose, we use the following global branch theorem established in [10].

Theorem 1.1: Let $X$ and $Y$ be real Banach spaces, $\mathcal{B}(X, Y)$ be thespace of bounded linear operators from $X$ into $Y$ with its usualnorm, $P: \mathbb{R} \times X \rightarrow \mathbb{R}$ denote theprojection $P(\lambda, u)=\lambda$, and

$\Phi_{0}(X, Y)=$

$\{L \in \mathcal{B}(X, Y): L$ is a Fredholm operator of index zero $\}$.

Let $L \in C^{1}(J, \mathcal{B}(X, Y))$ where $J$ is an open interval and $L(\lambda) \in \Phi_{0}(X, Y)$ for all $\lambda \in J$. Suppose that there exists $\lambda_{0} \in J$ suchthat dimker $L\left(\lambda_{0}\right)$ is odd and

$$
L^{\prime}\left(\lambda_{0}\right) \operatorname{ker} L\left(\lambda_{0}\right) \oplus \operatorname{reg} L\left(\lambda_{0}\right)=Y
$$

Let $K \in C(X, Y)$ be such that $K: X \rightarrow Y$ is compact and

$$
\lim _{\|u\|_{X} \rightarrow 0} \frac{\|K(u)\|_{Y}}{\|u\|_{X}}=0 \text {. }
$$

Let $\quad \tilde{Z}=Z \cup\left\{\left(\lambda_{0}, 0\right)\right\} \quad$ where $\quad Z=\{(\lambda, u) \in J \times X: u \neq$ 0 and $L(\lambda) u+K(u)=0\}$ be considered with the metric inherited from $\mathbb{R} \times X$, and let $\mathcal{C}$ denote the connected component of $\tilde{Z}$ containing $\left(\lambda_{0}, 0\right)$. Then $\mathcal{C}$ possesses at least one of the followingproperties:

(i) $\mathcal{C}$ is an unbounded subset of $\mathbb{R} \times X$;

(ii) $\overline{\mathcal{C}} \cap[J \times\{0\}] \neq\left\{\left(\lambda_{0}, 0\right)\right\}$, where $\overline{\mathcal{C}}$ is the closure of $\mathcal{C}$ in $J \times X$;

(iii) eithersupPC $=\operatorname{supJ}$ or infPC $=$ infJ.

REMARK 1.1:For $K \in C(X, Y)$, the condition (1.3) is equivalent to theproperties $K(0)=0$ and $K: X \rightarrow Y$ is FrÉchetdifferentiable at zero with $K^{\prime}(0)=0$.

By $\left(F_{1}\right)$ and $\left(F_{2}\right)$ we may define a function $k$ having the following properties

Identify applicable sponsor/s here. (sponsors) 


$$
k(x, s)=g(x)-\frac{f(x, s)}{s}
$$

with $\lim _{s \rightarrow 0} k(x, s)=g(x)-h(x), \lim _{|s| \rightarrow+\infty} k(x, s)=0$ uniformly in $x \in \mathbb{R}^{N}$ and $0 \leq k(x, s) \leq g(x)-h(x)$. From the above notation (1.4),problem(1.1)is equivalent to

$-\Delta u-g(x) u+\lambda V(x) u+k(x, u) u=0, u \in H^{1}\left(\mathbb{R}^{N}\right)(1.5) \mathrm{To}$ prove the asymptotic bifurcation result, first we study the following formal asymptotic linearization of (1.5):

$$
\left\{\begin{array}{c}
-\Delta u-g(x) u+\lambda V(x) u=0, \\
u \in H^{1}\left(\mathbb{R}^{N}\right), \lambda>0 .
\end{array}\right.
$$

A number $\lambda>0$ is said to be an eigenvalue of (1.6)if there exists $u \in H^{1}\left(\mathbb{R}^{N}\right) \backslash\{0\}$ such that

$\int_{\mathbb{R}^{N}}[\nabla u \nabla v-g(x) u v+\lambda V u v] d x=0$ for all $v \in H^{1}\left(\mathbb{R}^{N}\right)$.

For the discussion of equation (1.5), we take advantage ofthe additional regularity of solutions that follows from ourassumptions(see Proposition 2.1 in [10]).

Proposition 1.1: (1) Assume that the conditions $\left(F_{1}\right)\left(F_{2}\right)\left(V_{1}\right)\left(V_{2}\right)$ hold and $u \in H^{1}\left(\mathbb{R}^{N}\right)$ satisfies (1.5), then $u \in W^{2, p}\left(\mathbb{R}^{N}\right)$ for all $p \in[2,+\infty)$ andhence $u \in C^{1}\left(\mathbb{R}^{N}\right)$ with $\lim _{|x| \rightarrow+\infty} u(x)=0 \operatorname{and}_{|x| \rightarrow+\infty} \lim _{n} \nabla u(x)=0$.

(2) If $V$ satisfies $\left(V_{1}\right)\left(V_{2}\right)$ and $v \in H^{1}\left(\mathbb{R}^{N}\right)$ is an eigenfunction of (1.6), then $v \in W^{2, p}\left(\mathbb{R}^{N}\right)$ for all $p \in[2,+\infty)$.

Our first result concerning the linearized equation (1.6) is the following :

Theorem 1.2: Assume that $V$ and $g$ satisfies $\left(V_{1}\right)\left(V_{2}\right)$ and (G), then

(i) there exists an unique eigenvalue $\lambda=\Lambda(\alpha)$ of (1.6) having a positive eigenfunction. Furthermore $\Lambda(\alpha)>1$, and it is simple in the sense that $\operatorname{ker}\left(A_{\Lambda(\alpha)}\right)=\operatorname{span}\left\{u_{\Lambda(\alpha)}\right\}$ where $A_{\lambda}$ denotes the Schrö dinger operator $A_{\lambda} u=-\Delta u-g(x) u+$ $\lambda V u$ and $u_{\Lambda(\alpha)}>0$ on $\mathbb{R}^{N}$. All other eigenvalues of (1.6) are less than $\Lambda(\alpha)$ and their eigenfunctions change sign.

(ii) $\Lambda(\alpha)$ is the unique value of $\lambda$ in theinterval $\left[\frac{\alpha}{V(\infty)},+\infty\right)$ for which 0 is theinfimum of the spectrum of the Schrödinger operator $A_{\lambda}$.

Now we can state our main result concerning the nonlinear problem (1.1).

Theorem 1.3: Let the conditions $\left(F_{1}\right)\left(F_{2}\right)\left(F_{3}\right)\left(V_{1}\right)\left(V_{2}\right)$ hold and fix $P \in[2,+\infty) \cap\left(\frac{\mathrm{N}}{2},+\infty\right)$. Thenthere exist two connected subsets $\Sigma^{+}$and $\Sigma^{-}$of $\mathbb{R} \times W^{2, p}\left(\mathbb{R}^{N}\right)$, whoseelements $(\lambda, u)$ are, respectively, positive and negativesolutions ofproblem (1.1), such that $\inf \{\lambda:(\lambda, u) \in$ $\left.\Sigma^{ \pm}\right\}=$
$\frac{\alpha}{V(\infty)}$ and $u p\left\{\lambda:(\lambda, u) \in \Sigma^{ \pm}\right\}=\Lambda(\alpha)$, where $\Lambda(\alpha)$ is given by Theorem 1.2. Furthermore, $\Sigma^{ \pm}$is bounded away from the line of trivial solutions of $\mathbb{R} \times\{0\}$ and if $\left\{\left(\lambda_{n}, u_{n}\right)\right\} \subset$ $\Sigma^{ \pm}$with $\lambda_{n} \stackrel{n}{\rightarrow} \lambda>\frac{\alpha}{V(\infty)}$, then $\max _{x \in \mathbb{R}^{N}}\left|u_{n}(x)\right| \stackrel{n}{\rightarrow} \infty$ if and only if $\lambda=\Lambda(\alpha)$.

\section{EIGENVALUE PROBLEM}

In this section, we prove Theorem 1.2. It follows from Proposition 1.1 that anyeigenfunction $u$ of equation (1.6) belongs to $C\left(\mathbb{R}^{N}\right) \cap H^{2}\left(\mathbb{R}^{N}\right)$, and this leads us to introduce a Schrödinger operator having $u$ as an eigenfunction.Define $A_{\lambda}: D\left(A_{\lambda}\right)=H^{2}\left(\mathbb{R}^{N}\right) \subset L^{2}\left(\mathbb{R}^{N}\right) \rightarrow L^{2}\left(\mathbb{R}^{N}\right)$ by $A_{\lambda}=-\Delta u-g(x) u+\lambda V u$

Then $A_{\lambda}$ is a self-adjoint operator in $L^{2}\left(\mathbb{R}^{N}\right)$ with spectrum $\sigma\left(A_{\lambda}\right)$ and essential spectrum $\sigma_{e}\left(A_{\lambda}\right)=[\lambda V(\infty)-$ $\alpha,+\infty)$.Furthermore, setting $\sum(\lambda)=\inf \sigma\left(A_{\lambda}\right)$, we have $\sum(\lambda)=\inf \left\{a_{\lambda}(u): u \in H^{1}\left(\mathbb{R}^{N}\right)\right.$ and $\left.\int_{\mathbb{R}^{N}} u^{2} d x=1\right\}>$ $-\infty$, where $a_{\lambda}(u)=\int_{\mathbb{R}^{N}}\left[|\nabla u|^{2}-g(x) u^{2}+\lambda V u^{2}\right] d x$.

Lemma 2.1: Suppose that $V$ satisfies $\left(V_{1}\right)\left(V_{2}\right)$ and $\Gamma<\alpha$, then $\sum(1)<0$. Moreover, there exists $\lambda_{1}>1$ such that $\sum(\lambda)<$ 0 for all $\lambda \in\left(-\infty, \lambda_{1}\right]$.

proof: Since $\Gamma<\alpha$, there exists $u \in H^{1}\left(\mathbb{R}^{N}\right) \backslash\{0\}$ such that $\int_{\mathbb{R}^{N}}\left(|\nabla u|^{2}+V(x) u^{2}\right) d x<\alpha \int_{\mathbb{R}^{N}} u^{2} d x \leq$ $\int_{\mathbb{R}^{N}} g(x) u^{2} d x$. This means that $a_{1}(u)<0$ and $\sum(1)<0$. Hence there exists $u_{1} \in H^{1}\left(\mathbb{R}^{N}\right)$ with $\int_{\mathbb{R}^{N}} u_{1}^{2} d x=1$ such that $a_{1}\left(u_{1}\right)<0$. By thedefinition of $a_{\lambda}$, we have $a_{\lambda}\left(u_{1}\right)-a_{1}\left(u_{1}\right)=(\lambda-1) \int_{\mathbb{R}^{N}} V(x) u_{1}^{2} d x$ for all $\lambda \in \mathbb{R} .(2.1)$ By $\left(V_{1}\right)\left(V_{2}\right)$, we have there exists $C>0$ such that $V(x) \leq$ $C$ for all $x \in \mathbb{R}^{N}$. From (2.1), we have $a_{\lambda}\left(u_{1}\right) \leq a_{1}\left(u_{1}\right)+$ $C(\lambda-1)$. Therefore choosing $\lambda_{1}=1+\frac{-a_{1}\left(u_{1}\right)}{2 C}>1$, we get $a_{\lambda_{1}}\left(u_{1}\right) \leq \frac{a_{1}\left(u_{1}\right)}{2}<0$ and $\sum\left(\lambda_{1}\right)<0$. Since $\sum(\lambda)$ is increasing with respectto $\lambda \in \mathbb{R}$, we have $\sum(\lambda)<0$ for all $\lambda \in\left(-\infty, \lambda_{1}\right]$.

Lemma 2.2: Let $V$ satisfies $\left(V_{1}\right)\left(V_{2}\right)$. For $\Gamma<\alpha<V(\infty)$, if we set $S:=\left\{\lambda \geq \frac{\alpha}{V(\infty)}: \sum(\lambda)<0\right\}$ and $\Lambda(\alpha)=\operatorname{supis}(\lambda: \lambda \in S\}$, then $\Lambda(\alpha) \in(1,+\infty)$.

Proof: From Lemma 2.1, we have $\Lambda(\alpha)>1$. It is clear that $S$ is an interval since $\sum(\lambda)$ is increasing in $\lambda$. Therefore, if $\Lambda(\alpha)=+\infty$, we have $S=\left[\frac{\alpha}{V(\infty)},+\infty\right)$ and for any integer $n \geq \frac{\alpha}{V(\infty)}$, there exists $u_{n} \in H^{1}\left(\mathbb{R}^{N}\right)$ with $\int_{\mathbb{R}^{N}} u_{n}^{2} d x=1$ such that

$a_{n}\left(u_{n}\right)=\int_{\mathbb{R}^{N}}\left[\left|\nabla u_{n}\right|^{2}-g(x) u_{n}^{2}+n V(x) u_{n}^{2}\right] d x<0$.

By condition $\left(V_{1}\right)$ we see that (2.2) is impossible when $n \geq \frac{\alpha}{V_{0}}$.

Lemma 2.3: Assume that $\left(V_{1}\right)\left(V_{2}\right)$ hold and $\Gamma<\alpha<V(\infty)$. Then $\lambda \in\left[\frac{\alpha}{V(\infty)},+\infty\right)$ and $\sum(\lambda)=0$ if and only if $\lambda=\Lambda(\alpha)$, where $\Lambda(\alpha)$ is given by Lemma 2.2 . 
Proof: Since $\Gamma<\alpha<V(\infty)$ and $\sum(\lambda)$ is increasing in $\lambda$, it follows from Lemma2.1 that $\sum\left(\frac{\alpha}{V(\infty)}\right) \leq \sum(1)<0$. If $\lambda \in\left[\frac{\alpha}{V(\infty)},+\infty\right)$ and $\sum(\lambda)=0$, then $\lambda>\frac{\alpha}{V(\infty)}$. Nowwe have $\Sigma(\lambda)=\inf \sigma\left(A_{\lambda}\right)=0$ and inf $\sigma_{e}\left(A_{\lambda}\right)=\lambda V(\infty)-\alpha>$ $\frac{\alpha}{V(\infty)} V(\infty)-\alpha=0$. Hence 0 is aneigenvalue of $A_{\lambda}$ and there exists $u_{\lambda} \in C\left(\mathbb{R}^{N}\right) \cap H^{2}\left(\mathbb{R}^{N}\right)$ such that $\operatorname{ker} A_{\lambda}=\operatorname{span}\left\{u_{\lambda}\right\}$ and $u_{\lambda}>0$ on $\mathbb{R}^{N}$ (see [9], Theorem 3.20] for example). We mayassume that $\int_{\mathbb{R}^{N}} u_{\lambda}^{2} d x=1$ such that $a_{\lambda}\left(u_{\lambda}\right)=0$. Then from the definition of $a_{\lambda}$ we have for any $\varepsilon>0$

$$
a_{\lambda-\varepsilon}\left(u_{\lambda}\right)=-\varepsilon \int_{\mathbb{R}^{N}} V u_{\lambda}^{2} d x \leq-\varepsilon V_{0} \int_{\mathbb{R}^{N}} u_{\lambda}^{2} d x<0
$$

and thismeans that $\lambda-\varepsilon \in S$ for any $\varepsilon>0$. Therefore $\lambda$

$=\operatorname{supS}=\Lambda(\alpha)$.

Conversely, if $\lambda=\Lambda(\alpha)$, by Lemma 2.2 wehave $\Lambda(\alpha)>$ $1>\frac{\alpha}{V(\infty)}$. Hence it issufficient to $\operatorname{prove} \Lambda(\alpha) \notin S \cup T$, where $T$ $:=\left\{\lambda \geq \frac{\alpha}{V(\infty)}: \sum(\lambda)>0\right\}$. Indeed, if $\Lambda(\alpha) \in S$, then $\sum(\Lambda(\alpha))<0$. By the proof of Lemma 2.1 we see that there exists $\lambda_{2}>\Lambda(\alpha)$ such that $\sum(\lambda)<0$ for all $\lambda \in\left(-\infty, \lambda_{2}\right]$. This contradicts the definition of $\Lambda(\alpha)$. On the other hand, if $\Lambda(\alpha) \in$ $T$, then $\Sigma(\Lambda(\alpha))>0$. By the definition of $a_{\lambda}$ and $V(x) \leq C$ for all $x \in \mathbb{R}^{N}$, we see that for any $\varepsilon>0$ and $u \in H^{1}\left(\mathbb{R}^{N}\right)$ with $\int_{\mathbb{R}^{N}} u^{2} d x=1$,

$$
a_{\Lambda(\alpha)-\varepsilon}(u)=a_{\Lambda(\alpha)}(u)-\varepsilon \int_{\mathbb{R}^{N}} V u^{2} d x \geq \sum(\Lambda(\alpha))-\varepsilon C
$$

Therefore we can choose $\varepsilon=\frac{\Sigma(\Lambda(\alpha))}{2 C}$ such that $a_{\Lambda(\alpha)-\varepsilon}\left(u_{\lambda}\right) \geq \frac{\sum(\Lambda(\alpha))}{2}>0$ for all $u \in H^{1}\left(\mathbb{R}^{N}\right)$ with $\int_{\mathbb{R}^{N}} u^{2} d x=1$. This means that $\sum(\Lambda(\alpha)-\varepsilon)>0$ and also contradicts thedefinition of $\Lambda(\alpha)$.

Proof of Theorem 1.2 (i) From Lemma 2.2 and 2.3 we know that $\Lambda(\alpha)>1$ and $\sum(\Lambda(\alpha))=\inf \sigma\left(A_{\Lambda(\alpha)}\right)=0$. Since $\alpha<V(\infty)$, we have inf $\sigma_{e}\left(A_{\Lambda(\alpha)}\right)=\Lambda(\alpha) V(\infty)-\alpha>0$. Hence 0 is aneigenvalue of $A_{\Lambda(\alpha)}$ and there existsu $u_{\Lambda(\alpha)} \in$ $C\left(\mathbb{R}^{N}\right) \cap H^{2}\left(\mathbb{R}^{N}\right)$ such that $\operatorname{ker} A_{\Lambda(\alpha)}=\operatorname{span}\left\{u_{\Lambda(\alpha)}\right\}$ and $u_{\Lambda(\alpha)}>0$ on $\mathbb{R}^{N}$. Suppose now that $\Lambda_{1} \neq \Lambda(\alpha)$ is another eigenvalue of(1.6) with eigenfunction $u_{1} \in H^{1}\left(\mathbb{R}^{N}\right)$. Then 0 is an eigenvalue of $A_{\Lambda_{1}}$ and $\sum\left(\Lambda_{1}\right)=\inf \sigma\left(A_{\Lambda_{1}}\right) \leq 0$. It follows that $\Lambda_{1} \leq \Lambda(\alpha)$. Otherwise, if $\Lambda_{1}>\Lambda(\alpha)$ and $\Sigma\left(\Lambda_{1}\right) \leq 0$, we divide two cases to deduce thecontradiction. One hand, if $\Lambda_{1}>\Lambda(\alpha)$ and $\sum\left(\Lambda_{1}\right)=0$, it contradicts Lemma 2.3. On theother hand, if $\Lambda_{1}>\Lambda(\alpha)$ and $\sum\left(\Lambda_{1}\right)<0$, by the proof of Lemma 2.1 we see thatthere exists $\lambda_{3}>\Lambda_{1}$ such that $\sum(\lambda)<$ 0 for all $\lambda \in\left(-\infty, \lambda_{3}\right]$. This contradicts thedefinition of $\Lambda(\alpha)$. Therefore $\Lambda(\alpha)$ isthe largest eigenvalue of (1.6). Furthermore, integratingby parts, we have

$$
\left(\Lambda(\alpha)-\Lambda_{1}\right) \int_{\mathbb{R}^{N}} V u_{1} u_{\Lambda(\alpha)} d x=0 .
$$

For $\Lambda_{1}<\Lambda(\alpha)$ and $V(x) u_{\Lambda(\alpha)}>0$ on $\mathbb{R}^{N}$, it follows that $u_{1}$ changes sign.

(ii) This follows from Lemma 2.3.

\section{THE TRUNCATED PROBLEM}

Let $P \in[2,+\infty) \cap\left(\frac{N}{2},+\infty\right)$ be fixed and we set $X=W^{2, p}\left(\mathbb{R}^{N}\right)$ with $\|\cdot\|=\|\cdot\|_{W^{2, p}\left(\mathbb{R}^{N}\right)}$, and

$Y=L^{p}\left(\mathbb{R}^{N}\right)$ with $|\cdot|_{p}=\|\cdot\|_{L^{p}\left(\mathbb{R}^{N}\right)}$.

For $k$ defined in (1.4), it can be shown that (see [10], Lemma B.1] )

$$
\frac{|k(x, u) u|_{p}}{\|u\|} \nrightarrow 0 \text { as }|| u|| \rightarrow+\infty
$$

uniformly in $x \in \mathbb{R}^{N}$. Hence in order to make use ofTheorem 1.1 we need to introduce the following truncatedproblem

$$
-\Delta u-g(x) u+\lambda V(x) u+\psi_{n}(x) k(x, u) u=0
$$

where $u \in H^{1}\left(\mathbb{R}^{N}\right)$ and

$$
\psi_{n}(x)=\left\{\begin{array}{lll}
1, & \text { if } & |x| \leq n \\
0, & \text { if } \quad|x|>n
\end{array}\right.
$$

Define $L(\lambda): X \rightarrow Y$ by

$$
L(\lambda) u=-\Delta u-g(x) u+\lambda V(x) u
$$

Using the inversion, $u \mapsto v=\frac{u}{\|\left. u\right|^{2}}$, problem (3.1) is equivalent to

$$
L(\lambda) v+K_{n}(v)=0
$$

where

$$
K_{n}(v)=\left\{\begin{array}{cl}
\psi_{n} k\left(x, \frac{v}{\|v\|^{2}}\right) v, & \text { for } v \in X \backslash\{0\} \\
0, & \text { for } v \equiv 0
\end{array}\right.
$$

In the sequel we show that Theorem 1.1 is applicable tothe inverted truncated problem (3.3). First, it follows fromTheorem 4.3 of [6] that $L(\lambda) \in \Phi_{0}(X, Y)$ for all $>\frac{\alpha}{V(\infty)}$, where $\Phi_{0}(X, Y)$ is defined in Theorem 1.1 and

$$
\mathrm{Y}=\operatorname{ker} L(\lambda) \oplus \operatorname{rge} L(\lambda) \text { for all } \lambda>\frac{\alpha}{V(\infty)}
$$

From (3.4) and Theorem1.2 we can prove (1.2) holds with $\lambda_{0}=\Lambda(\alpha)$ (see the proof of Theorem 4.2in [10]). By Remark 1.1 the following lemma(Lemma 3.2 in [10]) can verify condition (1.3) for $K_{n}$ defined in(3.3) .

Lemma 3.1: For all $n \in N, K_{n} \in C(X, Y) \cap C^{1}(X \backslash\{0\}, Y)$, $K_{n}: X \rightarrow Y$ is compact and it is Fréchetdifferentiable at 0 with $K_{n}^{\prime}(0)=0$.

Now we can apply Theorem 1.1 for $L(\lambda)$ defined by (3.2) on the interval $J=\left(\frac{\alpha}{V(\infty)},+\infty\right)$ and at the point $\lambda_{0}=\Lambda(\alpha)$. Set $Z_{n}=\left\{(\lambda, v) \in\left(\frac{\alpha}{V(\infty)},+\infty\right) \times X: v\right.$ is a nontrivial solution of 
(3.3)\}. Let $\mathcal{C}_{n}$ denote the connected component of $\mathcal{Z}_{n} \cup$ $\{(\Lambda(\alpha), 0)\}$ containing $(\Lambda(\alpha), 0)$. In order to get some initial informationabout $\mathcal{C}_{n}$ from Theorem 1.1, we establish someestimates about the solutions of (3.2) that will help us toobtain more precise information about $\mathcal{C}_{n}$.

Lemma 3.2: Let the conditions $\left(V_{1}\right)\left(V_{2}\right)$ hold and $\alpha=l+$ $\mu$ Suppose that $\Gamma<\alpha<V(\infty)$ and $0 \leq \mu<\frac{l V_{0}}{V(\infty)-V_{0}}$. Then, there exists $T>0$, such that for any $\beta>\frac{\alpha}{V(\infty)}$ there is $N_{\beta} \in$ $\mathbb{N}$ satisfying $\|v\| \leq T$ for all $(\lambda, v) \in Z_{n}$ with $\lambda \geq \beta$ and $n \geq N_{\beta}$.

Proof :Firstwe define the bound $T$. Since $0 \leq \mu<\frac{l V_{0}}{V(\infty)-V_{0}}$ and $\alpha=l+\mu$, we candeduce that $\mu<\frac{\alpha V_{0}}{V(\infty)}$ and choose $\eta=$ $\frac{1}{2}\left(\frac{\alpha V_{0}}{V(\infty)}-\mu\right)>0$. By (1.4) and $\left(V_{1}\right)$ we know $k(x, 0)=l$ and there exists $\delta_{1}$ such that $k(x, s) \geq l-\eta=\frac{1}{2} l+\frac{1}{2}\left(\alpha-\frac{\alpha V_{0}}{V(\infty)}\right) \geq$ $\frac{1}{2} l>0$ for all $|s| \leq \delta_{1}$. By Sobolevembeding there is $C_{1}$ suchthat $|u|_{\infty} \leq C_{1}|| u||$. Set $T=\frac{C_{1}}{\delta_{1}}$. Suppose that $v \in X$ and ||$v|| \geq T$. Then, for all $x \in \mathbb{R}^{N}$, we have $\frac{|v(x)|}{|| v||^{2}} \leq \frac{|v|_{\infty}}{\left.|| v\right|^{2}} \leq \frac{C_{1}}{|| v||} \leq$ $\frac{C_{1}}{\mathrm{~T}}=\delta_{1}$. Consequently, $k\left(x, \frac{|v(x)|}{\left.|| v\right|^{2}}\right) \geq l-\eta$. For any $\beta>\frac{\alpha}{V(\infty)}$, we can choose $\delta_{\beta}=V(\infty)-\frac{\alpha}{\beta}>0$. By $\left(V_{2}\right)$, there $\operatorname{exists} N_{\beta} \in$ $\mathbb{N}$, such that $V(x)=V(\infty)-\delta_{\beta}$ for all $|x| \geq N_{\beta}$. Hence

$$
\lambda \mathrm{V}(\mathrm{x}) \geq \beta\left(\mathrm{V}(\infty)-\delta_{\beta}\right)=\alpha
$$

for all $\lambda \geq \beta$ and $|x| \geq N_{\beta}$. On the otherhand, for $\lambda \geq \beta$, $n \geq N_{\beta},|x| \leq N_{\beta}$, and ||$v|| \geq T$, we have

$$
K_{n}(v)=\psi_{n} k\left(x, \frac{|v|}{|| v||^{2}}\right)=k\left(x, \frac{|v|}{\|v\|^{2}}\right) \geq l-\eta,
$$

and $-\alpha+\lambda V(x)+K_{n}(v) \geq-\alpha+\frac{\alpha}{V(\infty)} V_{0}+l-\eta$

$$
=-\mu-\eta+\frac{\alpha}{V(\infty)} V_{0}=\frac{1}{2}\left(\frac{\alpha V_{0}}{V(\infty)}-\mu\right)>0 .
$$

Combining the above inequality and (3.5), we get that for $(\lambda, v) \in Z_{n}$ with $\lambda \geq \beta$ and $n \geq N_{\beta}$, if ||$v|| \geq T$, then

$$
v(x) \Delta v(x)=v^{2}(x)\left[-\alpha+\lambda V(x)+\psi_{n} k\left(x, \frac{|v|}{\left.|| v\right|^{2}}\right)\right] \geq 0
$$

for all $x \in \mathbb{R}^{N}$. The maximum principle now leads toa contradiction.

Lemma 3.3: Fix $\beta>\frac{\alpha}{V(\infty)}$. Then, for any $\varepsilon \in\left(0, V(\infty)-\frac{\alpha}{\beta}\right)$, there exists $C_{\varepsilon}>0$ such that $|u(x)| \leq|u|_{\infty} e^{-\sqrt{\xi}\left(|x|-C_{\varepsilon}\right)}$ for all $x \in \mathbb{R}^{N}$,where $\xi=\beta(V(\infty)-\varepsilon)-\alpha>0$ for all $(\lambda, u) \in$ $[\beta,+\infty)$ with $u L(\lambda) u \leq 0$ on $\mathbb{R}^{N}$.
Proof: $\quad$ Since $\lim _{|x| \rightarrow+\infty} V(x)=V(\infty)$, for any $\varepsilon \in(0, V(\infty)-$ $\left.\frac{\alpha}{\beta}\right)$, there exists $C_{\varepsilon}>0$ suchthat $V(x) \geq V(\infty)-\varepsilon$ for all $|x| \geq C_{\varepsilon}$. Set $q(x)=|u|_{\infty} e^{-\sqrt{\xi}\left(|x|-C_{\varepsilon}\right)}-u(x)$ and $\Omega_{\varepsilon}=$ $\left\{x \in \mathbb{R}^{N}:|x|>C_{\varepsilon}\right.$ and $\left.q(x)<0\right\}$. For all $x \in \Omega_{\varepsilon}$, we have $u(x)>0$ and

$$
\begin{aligned}
& 0 \geq L(\lambda) u=-\Delta u-\alpha u+\lambda V(x) u \\
& \geq-\Delta u-\alpha u+\beta(V(\infty)-\varepsilon) u=-\Delta u+\xi u,
\end{aligned}
$$

since $\geq \beta$. By direct calculations, we have for $x \in \Omega_{\varepsilon}$

$$
\begin{gathered}
\Delta q(x)=|u|_{\infty} e^{\sqrt{\xi} C_{\varepsilon}}\left(\xi-\frac{N-1}{|x|} \sqrt{\xi}\right) e^{-\sqrt{\xi}|x|}-\Delta u \\
\leq|u|_{\infty} e^{\sqrt{\xi} C_{\varepsilon}} \xi e^{-\sqrt{\xi}|x|}-\xi u=\xi q(x)<0 .
\end{gathered}
$$

Since $q(x) \rightarrow 0$ as $|x| \rightarrow+\infty$ and $q(x) \geq 0$ for $|x|=C_{\varepsilon}$, we have $q(x) \geq 0$ for $x \in \Omega_{\varepsilon}$. If $\Omega_{\varepsilon} \neq \emptyset$, the weakmaximum principle (Theorem 8.1 in [4]) implies that $q(x) \geq 0$ in $\Omega_{\varepsilon}$, a contradiction. Thus we see that $|u(x)| \leq|u|_{\infty} e^{-\sqrt{\xi}\left(|x|-C_{\varepsilon}\right)}$ for all $|x| \geq C_{\varepsilon}$. Replacing $u$ by $-u$, we get the above inequality for $|u(x)|$. Hence, we complete the proof.

Lemma 3.4: For each $n \in \mathbb{N}$, there exists an open neighborhood $U$ of $(\Lambda(\alpha), 0) \in \mathbb{R} \times X$, such that $u^{2}>0$ on $\mathbb{R}^{N}$ for all $(\lambda, v) \in U \cap Z_{n}$.

Proof: By contradiction, suppose that there exists asequence $\left\{\left(\lambda_{i}, u_{i}\right)\right\} \subset Z_{n}$ such that $\lambda_{i} \stackrel{i}{\rightarrow} \Lambda(\alpha)$ and $\|u\|_{i} \stackrel{i}{\rightarrow} 0$ andeach continuous function $u_{i}$ has at least one zero in $\mathbb{R}^{N}$. We prove this leadsto a contradiction.

Setting $z_{i}=\frac{u_{i}}{\|u\|_{i}}$, by the definition of $Z_{n}$, we have $L\left(\lambda_{i}\right) z_{i}+\frac{K_{n}\left(u_{i}\right)}{\|u\|_{i}}=0$ on $\mathbb{R}^{N}$. Since $V(x) \in L^{\infty}\left(\mathbb{R}^{N}\right)$, by Lemma 3.1, wefind that

$$
L(\Lambda(\alpha)) z_{i}=\left(\Lambda(\alpha)-\lambda_{i}\right) V(x) z_{i}-\frac{K_{n}\left(u_{i}\right)}{\|u\|_{i}} \stackrel{i}{\rightarrow} 0 \text { in } Y .
$$

On the other hand, by passing to a subsequence, we may supposethat $z_{i} \rightarrow z$ weakly in $X$. Since $\Lambda(\alpha)>1>\frac{\alpha}{V(\infty)}$, we have $L(\Lambda(\alpha)) \in \Phi_{0}(X, Y)$. By Lemma 3.5 of [10], we know that $z_{i} \stackrel{i}{\rightarrow} z \quad$ strongly in $X \quad$. This means that ||$z||=1$ and $L(\Lambda(\alpha)) z_{i}=0$. By Lemma3.3, wehave $z \in H^{1}\left(\mathbb{R}^{N}\right)$. According to Theorem 1.2(i), we may as well suppose that $\mathrm{Z}>0$ on $\mathbb{R}^{N}$. Since $\lambda_{i} \stackrel{i}{\rightarrow} \Lambda(\alpha)$ and $\Lambda(\alpha)>1$, choosing $\varepsilon=\frac{\Lambda(\alpha)-1}{2}>0$, there exists $i_{0} \in \mathbb{N}$ such that $\lambda_{i} \geq 1+$ $\varepsilon$ for all $i \geq i_{0}$. By $\left(V_{2}\right)$ and $\alpha<V(\infty)$, thereexists $R \geq n$ such that $V(x) \geq \alpha$ for all $|x| \geq R$. Hence, for all $i \geq i_{0}$ and $|x| \geq R$, we have $-\alpha+\lambda_{i} V(x) \geq-\alpha+(1+\varepsilon) \alpha>0$. Since $R \geq n$, we have for $|x|>R, \psi_{n}(x)=0$ and

$$
0=L\left(\lambda_{i}\right) z_{i}=-\Delta z_{i}+\left(-\alpha+\lambda_{i} V(x)\right) z_{i}
$$


Recalling that $X \subset C\left(\mathbb{R}^{N}\right)$, we have $\delta=\inf _{|x| \leq R} Z(x)>0$. Since $z_{i} \stackrel{i}{\rightarrow} z$ strongly in $X$, we have that $\underset{|x| \leq R}{\operatorname{Max} \mid z} z_{i}(x)-z(x) \mid \stackrel{i}{\rightarrow} 0$ and there exists $i_{1} \geq i_{0}$ such that $z_{i}(x) \geq \frac{1}{2} \delta$ for all $|x| \leq R$ and $i \geq i_{1}$. Since $z_{i}(x) \geq \frac{1}{2} \delta$ for $|x|=R$ and $\lim _{|x| \rightarrow+\infty} Z_{i}(x)=0$, we get $z_{i}(x) \geq 0$ on $\partial B_{R}^{c}$. Now we can apply the strong maximumprinciple (Theorem 8.19 in [4]) to equation (3.6) on theregion $B_{R}^{c}$ and deduce that $z_{i}>0$ in $B_{R}^{c}$ for alli $\geq i_{1}$. This contradicts that each $u_{i}$ has at least onezero in $\mathbb{R}^{N}$.

Now we can give more information about $\mathcal{C}_{n}$.

Theorem 3.1: Let $\mathcal{C}_{n}$ denote the connected component of $\mathcal{Z}_{n} \cup\{(\Lambda(\alpha), 0)\}$ containing the $\operatorname{point}(\Lambda(\alpha), 0)$. Then:

(i) $u^{2}>0$ on $\mathbb{R}^{N}$ and $\lambda \leq \Lambda(\alpha)$ for all $(\lambda, u) \in$ $\mathcal{C}_{n} \backslash\{(\Lambda(\alpha), 0)\}$;

(ii) for any $\beta>\frac{\alpha}{V(\infty)}$, there exist $T>0$ and $N_{\beta} \in \mathbb{N}$ such that, for all $n \geq N_{\beta}$,

$\operatorname{infP\mathcal {C}_{n}}:=\quad \inf \left\{\lambda:(\lambda, u) \in \mathcal{C}_{n}\right\}<\beta$ and $\|u\| \leq T$

for all $(\lambda, v) \in \mathcal{C}_{n}$ with $\lambda \geq \beta$.

Proof : The first step is to show that if $(\lambda, u) \in$ $\mathcal{C}_{n} \backslash\{(\Lambda(\alpha), 0)\}$, we have $\lambda \leq \Lambda(\alpha)$. Since $(\lambda, u)$ solves equation (3.3), we have

$$
-\Delta u-\alpha u+\lambda V(x) u+\psi_{n}(x) k\left(x, \frac{u}{\|u\|^{2}}\right) u=0
$$

By Lemma 3.3, we know $u \in H^{1}\left(\mathbb{R}^{N}\right)$. Hence byTheorem1.2 (ii) and (3.7),

$$
\begin{aligned}
& 0=\inf \left\{\int_{\mathbb{R}^{N}}\left[|\nabla v|^{2}-\alpha v^{2}+\Lambda(\alpha) V v^{2}\right] d x: v \in H^{1}\left(\mathbb{R}^{N}\right)\right\} \\
& \leq \int_{\mathbb{R}^{N}}\left[|\nabla u|^{2}-\alpha u^{2}+\Lambda(\alpha) V u^{2}+\psi_{n}(x) k\left(x, \frac{|u|}{\left.|| u\right|^{2}}\right) u^{2}\right] d x \\
& =(\Lambda(\alpha)-\lambda) \int_{\mathbb{R}^{N}} V(x) u^{2} d x .
\end{aligned}
$$

Since $\int_{\mathbb{R}^{N}} V(x) u^{2} d x \geq V_{0} \int_{\mathbb{R}^{N}} u^{2} d x>0$, we see that $\lambda \leq$ $\Lambda(\alpha)$.

The next step is to show that if $(\lambda, u) \in \mathcal{C}_{n} \backslash\{(\Lambda(\alpha), 0)\}$, then $u^{2}>0$ on $\mathbb{R}^{N}$. Set

$$
\mathcal{Q}=\left\{(\lambda, u) \in \mathcal{C}_{n}: u^{2}>0 \text { on } \mathbb{R}^{N}\right\} \cap\{(\Lambda(\alpha), 0)\} .
$$

We prove that $\mathcal{Q}$ is both anopen and closed subset of $\mathcal{C}_{n}$, then by theconnectedness of $\mathcal{C}_{n}$ we have $\mathcal{Q}=\mathcal{C}_{n}$.
First we prove that $\mathcal{Q}$ is open in $\mathcal{C}_{n}$. $\operatorname{Given}(\lambda, u) \in \mathcal{Q}$, we show that there exists an openneighborhood $U$ of $(\lambda, u)$ in $\mathbb{R}^{N} \times X$ suchthat $U \cap \mathcal{C}_{n} \subset \mathcal{Q}$. For $(\lambda, u)=(\Lambda(\alpha), 0)$ this is established in Lemma 3.4. $\operatorname{For}(\lambda, u) \in \mathcal{C}_{n}$ with $u^{2}>0$ on $\mathbb{R}^{N}$, wehave that $u$ does not change sign since $X \subset C\left(\mathbb{R}^{N}\right)$. We suppose that $u>0$ on $\mathbb{R}^{N}$, the case $u<0$ beingsimilar. By $\left(V_{2}\right)$ and $\lambda>\frac{\alpha}{V(\infty)}$, there exist $r>0$ and $R \geq n$ such that for all $\eta$ with $|\lambda-\eta| \leq r,-\alpha+\eta V(x)>0$ for all $|x| \geq R$.Let $\delta=\inf _{|x| \leq R} u(x)$. Then, $\delta>0$ andthere exists an open neighborhood $U$ of $(\lambda, u)$ in $\mathbb{R}^{N} \times X$ such that $|\lambda-\eta| \leq r$ and $_{|x| \leq R} \inf _{f} v(x) \geq \frac{\delta}{2}$ for all $(\eta, v) \in U$. Since $R \geq n$, for $|x|>R$, we have $\psi_{n}(x)=0$ andfor $(\eta, v) \in U \cap Z_{n}, L(\eta) v=-\Delta v+$ $(-\alpha+\eta V(x)) v=0$.As the proof of Lemma 3.4, the maximum principle impliesthat $v(x)>0$ for $|x|>R$ and then $v>0$ on $\mathbb{R}^{N}$. Hence $U \cap \mathcal{C}_{n} \subset \mathcal{Q}$ and $\mathcal{Q}$ is open.

Now we show that $\mathcal{Q}$ is closed in $\mathcal{C}_{n}$. Supposethat $(\lambda, u) \in$ $\mathcal{C}_{n}$ and there exists a sequence $\left\{\left(\lambda_{i}, u_{i}\right)\right\} \subset \mathcal{Q}$ such that $\lambda_{i} \stackrel{i}{\rightarrow} \lambda$ and $\left\|u_{i}-u\right\| \stackrel{i}{\rightarrow} 0$. If $u=0$, we must have $\lambda=\Lambda(\alpha)$ since $\mathcal{C}_{n} \cap[\mathbb{R} \times\{0\}]=\{(\Lambda(\alpha), 0)\}$ and so $(\lambda, u) \in \mathcal{Q}$. If $u \neq 0$, passing to a subsequence, we may as wellsuppose that $u_{i}>0$ on $\mathbb{R}^{N}$ for all $i \in \mathbb{N}$. Itfollows that $u \geq 0$ on $\mathbb{R}^{N}$ and $-\Delta u+$ $c_{+} u=c_{-} u \geq 0 \quad$ on $\quad \mathbb{R}^{N}$, where $c(x)=-\alpha+\lambda V(x)+$ $+\psi_{n} k\left(x, \frac{|u|}{\|u\|^{2}}\right)$. Bythe strong maximum principle we have $u>0$ on $\mathbb{R}^{N}$. Hence $(\lambda, u) \in \mathcal{Q}$ and $\mathcal{Q}$ is closed in $\mathcal{C}_{n}$.

Now we know that $\mathcal{Q}=\mathcal{C}_{n}$. We claim that thismeans that case (ii) in Theorem 1.1 cannot occur. Indeed, if $\mathcal{C}_{n}$ has the property (ii), there exist $\lambda \in J \backslash\{\Lambda(\alpha)\}$ and a sequence $\left\{\left(\lambda_{i}, u_{i}\right)\right\} \subset \mathcal{C}_{n}$ such that $\lambda_{i} \stackrel{i}{\rightarrow} \lambda$ and $\left\|u_{i}\right\| \stackrel{i}{\rightarrow} 0$. Setting $z_{i}=\frac{u_{i}}{\left\|u_{i}\right\|}$ and arguing as in the proof of Lemma 3.4, we may assume that $z_{i} \stackrel{i}{\rightarrow} z$ strongly in $X$ and $L(\lambda) z=0$ with ||$z||=1$. By Lemma 3.3, we know $z \in H^{1}\left(\mathbb{R}^{N}\right)$. It follows fromTheorem 1.2 (i) that $\lambda<\Lambda(\alpha)$ and $z$ changes sign on $\mathbb{R}^{N}$. On the other hand, since $\mathcal{Q}=\mathcal{C}_{n}$, the sequence $z_{i}$ can be chosen sothat $z_{i}>0$ on $\mathbb{R}^{N}$. Then we have $z \geq 0$ on $\mathbb{R}^{N}$ and this contradicts the earlier conclusion.

For any $\beta>\frac{\alpha}{V(\infty)}$, by Lemma 3.2 there exists $N_{\beta} \in \mathbb{N}$ such that ||$v|| \leq T$ for all $(\lambda, v) \in \mathcal{C}_{n}$ with $\lambda \geq \beta$ and $n \geq N_{\beta}$. Thus, if $n \geq N_{\beta}$ and $\operatorname{infP} \mathcal{C}_{n} \geq \beta$, we deduce that $\mathcal{C}_{n}$ is boundedin $\mathbb{R} \times X$. Since we have shown that $\mathcal{C}_{n}$ has not the property (ii) in Theorem 1.1, we must have $\mathcal{C}_{n}$ satisfying infP $\mathcal{C}_{n}=$ infJ $=$ $\frac{\alpha}{V(\infty)}$. This contradicts our earlier assumptioninf $P \mathcal{C}_{n} \geq \beta>$ $\frac{\alpha}{V(\infty)}$ and we complete the proof.

\section{PROOF OF THEOREM 1.3}

In this section, by using the globalbifurcation result for the inverted truncated problem (3.3), we first prove the bifurcation result for the following invertedproblem 


$$
L(\lambda) v+K(v)=0
$$

Where $K(v)=\left\{\begin{array}{cl}k\left(x, \frac{|v|}{|| v \|^{2}}\right) v, & \text { for } v \in X \backslash\{0\} \\ 0, & \text { for } v \equiv 0 .\end{array}\right.$

Set $\mathcal{Z}=\left\{(\lambda, v) \in\left(\frac{\alpha}{V(\infty)},+\infty\right) \times X\right.$ : vis a nontrivial solution of (4.1) $\}$ and $\tilde{Z}=\left\{(\lambda, v) \in Z: v^{2}>0\right.$ on $\left.\mathbb{R}^{N}\right\}$.

Lemma 4.1:Let $\left\{\left(\lambda_{n}, u_{n}\right)\right\} \subset \tilde{z}$ be a sequencesuch that $\lambda_{n} \stackrel{n}{\rightarrow} \lambda>\frac{\alpha}{V(\infty)}$ and $\left\|v_{n}\right\| \stackrel{n}{\rightarrow} 0$. Then $\lambda=\Lambda(\alpha)$.

Proof: First we show that

$$
\frac{\left|K\left(\frac{\left|v_{n}\right|}{\|\left. v_{n}\right|^{2}}\right)\right|_{p}}{\left\|v_{n}\right\|} \stackrel{n}{\rightarrow} 0
$$

for all $p \in(1,+\infty)$. Since $\left(\lambda_{n}, v_{n}\right)$ solves(4.1), we have $v_{n} L\left(\lambda_{n}\right) v_{n} \leq 0$ on $\mathbb{R}^{N}$. From $\lambda_{n} \stackrel{n}{\rightarrow} \lambda>\frac{\alpha}{V(\infty)}$, we may choose $\beta=\frac{\alpha}{V(\infty)}+\frac{1}{2}\left(\lambda-\frac{\alpha}{V(\infty)}\right)>\frac{\alpha}{V(\infty)}$ such that $\lambda_{n} \geq \beta$ for $n$ large. It follows from Lemma 3.3 that there exist $L>0$ and $\gamma>0$ which areindependent of $n$ such that

$$
\left|v_{n}(x)\right| \leq L\left|v_{n}\right|_{\infty} e^{-\gamma|x|} \text { for all } x \in \mathbb{R}^{N} .
$$

By the Sobolev embedding, there is $C>0$ which is independent of $n$ such that $\left|v_{n}\right|_{\infty} \leq C|| v_{n}||$. Thus, we have

$$
\frac{\left|K\left(\frac{\left|v_{n}\right|}{\|\left. v_{n}\right|^{2}}\right)\right|}{|| v_{n} \|} \leq L C e^{-\gamma|x|} \text { for all } x \in \mathbb{R}^{N} .
$$

Conseuqently, for every fixed $x \in \mathbb{R}^{N}$,

$$
\frac{\left|K\left(\frac{\left|v_{n}\right|}{|| v_{n} \|^{2}}\right)\right|}{\left\|v_{n}\right\|} \stackrel{n}{\rightarrow} 0
$$

Combining (4.3) and (4.5), it follows from the dominatedconvergence that (4.2) holds. Let $\omega_{n}=\frac{v_{n}}{\left\|v_{n}\right\|}$. Since $v_{n}^{2}>0$ on $\mathbb{R}^{N}$, passing to a subsequence, we mayassume that $\omega_{n}>0$ on $\mathbb{R}^{N}$ for all $n \in \mathbb{N}$ and for some $\omega \in X$ with $\omega \geq 0$ on $\mathbb{R}^{N}, \omega_{n} \rightarrow \omega$ weakly in $X$. By (4.2) wededuce that

$$
L(\lambda) \omega_{n}=L\left(\lambda_{n}\right) \omega_{n}-\left(\lambda_{n}-\lambda\right) V(x) \omega_{n} \stackrel{n}{\rightarrow} 0 \text { in } Y
$$

Since $\lambda>\frac{\alpha}{V(\infty)}$, we have $L(\lambda) \in \Phi_{0}(X, Y)$. By Lemma 3.5 of [1] we know that $\omega_{n} \stackrel{n}{\rightarrow} \omega$ strongly in $X$. So $L(\lambda) \omega=0$ and ||$\omega||=1$. By Lemma 3.3 we have $\omega \in H^{1}\left(\mathbb{R}^{N}\right)$. This means that $\lambda$ is an eigenvalue of equation (1.6) and its correspondingeigenfunction $\omega$ does not change sign. Thus it follows from Theorem 1.2 (i) that $\lambda=\Lambda(\alpha)$.

Theorem 4.1: Let $\mathcal{C}$ denote the connected component of $\tilde{Z} \cup\{(\Lambda(\alpha), 0)\}$ containing $\{(\Lambda(\alpha), 0)\}$. The following hold. (i) $\mathcal{C}$ is bounded with infPC $=\frac{\alpha}{V(\infty)}$ and $\sup P \mathcal{C}=\Lambda(\alpha)$.

(ii) If $\left\{\left(\lambda_{n}, v_{n}\right)\right\} \subset \mathcal{C}$ with $_{n \rightarrow+\infty} \lim _{n}=\Lambda(\alpha)$, then $\lim _{n \rightarrow+\infty}\left\|v_{n}\right\|=$ 0 .

Proof: (i) First we show that if $(\lambda, v) \in \tilde{z}$, then $\lambda<\Lambda(\alpha)$. Since $v \neq 0$ solves equation (4.1), by Lemma3.3, we know $v \in$ $H^{1}\left(\mathbb{R}^{N}\right)$ and then we deduce that $u=\frac{v}{\|v\|^{2}}$ solves equation (1.5). By Theorem 1.2 (ii) we have

$$
\begin{array}{r}
0=\inf \left\{\int_{\mathbb{R}^{N}}\left[|\nabla \omega|^{2}-\alpha \omega^{2}+\lambda V \omega^{2}\right] d x: \omega\right. \\
\left.\left.\qquad H^{1}\left(\mathbb{R}^{N}\right) \text { and } \int_{\mathbb{R}^{N}} \omega^{2} d x=1\right\}\right\}
\end{array}
$$

$$
\leq \frac{1}{\int_{\mathbb{R}^{N}} u^{2} d x}\left[(\Lambda(\alpha)-\lambda) \int_{\mathbb{R}^{N}} V(x) u^{2} d x-\int_{\mathbb{R}^{N}} k(x, u) u^{2} d x\right]
$$

$\operatorname{Thus}(\Lambda(\alpha)-\lambda) \int_{\mathbb{R}^{N}} V(x) u^{2} d x \geq \int_{\mathbb{R}^{N}} k(x, u) u^{2} d x$. We claim that $\int_{\mathbb{R}^{N}} k(x, u) u^{2} d x>0$. Indeed, since $\lim _{s \rightarrow 0} k(x, s)=l>0$ uniformly in $x \in \mathbb{R}^{N}$,there exists $\delta>0$ such that $k(x, s) \geq \frac{l}{2}$ for all $|s| \leq \delta$ and $x \in \mathbb{R}^{N}$. ByProposition 1.1 we have $\lim _{|x| \rightarrow+\infty} u(x)=0$ and there exists $R>0$ such that $|u(x)| \leq$ $\delta$ for $|x| \geq R$. Since $(\lambda, v) \in \tilde{Z}$, we have $v^{2}>0$ on $\mathbb{R}^{N}$ and $u^{2}>0$ on $\mathbb{R}^{N}$. Thereforewe deduce that

$$
\int_{\mathbb{R}^{N}} k(x, u) u^{2} d x \geq \int_{|x| \geq R} k(x, u) u^{2} d x \geq \frac{l}{2} \int_{|x| \geq R} u^{2} d x .
$$

It follows that $\lambda<\Lambda(\alpha)$. Hence $\{\lambda:(\lambda, v) \in \tilde{\mathcal{C}} \backslash\{(\Lambda(\alpha), 0)\} \subset$ $\left(\frac{\alpha}{V(\infty)}, \Lambda(\alpha)\right)$ and $\operatorname{supPC}=\Lambda(\alpha)$.

Secondly we show that ||$v \| \mid \leq T$ for all $(\lambda, v) \in \tilde{z}$, where $T>0$ is defined in Lemma 3.2. Bycontradiction, if $(\lambda, v) \in$ $Z$ and ||$v|| \geq T$, similar to the proof of Lemma 3.2, we have for all $x \in \mathbb{R}^{N}, K\left(\frac{v(x)}{|| v||^{2}}\right) \geq l-\eta$, where $\eta=\frac{1}{2}\left(\frac{\alpha V_{0}}{V(\infty)}-\mu\right)>0$. Consequently,

$$
\begin{gathered}
v \Delta v=\left[-\alpha+\lambda V(x)+k\left(x, \frac{v(x)}{\|v\|^{2}}\right)\right] v^{2}(x) \\
\geq\left[-\mu+\frac{\alpha V_{0}}{V(\infty)}-\eta\right] v^{2}(x)=\eta v^{2}(x) \geq 0 .
\end{gathered}
$$

The maximum principle leads to a contradiction. Therefore we seethat $\tilde{Z}$ is bounded in $\mathbb{R} \times X$ and sois $\mathcal{C}$. 
The next step is to prove that infPC $=\frac{\alpha}{V(\infty)}$. By contradiction, supposethat $\rho:=\inf P \mathcal{C}>\frac{\alpha}{V(\infty)}$. For any $\bar{\rho} \in$ $\left(\frac{\alpha}{V(\infty)}, \rho\right)$, we $\operatorname{set} A=\{(\lambda, v) \in \mathcal{Q}: \lambda \geq \bar{\rho}\}, A_{1}=\{(\Lambda(\alpha), 0)\}$, $A_{2}=\{(\lambda, v) \in \mathcal{Q}: \lambda=\bar{\rho}\}$, where $\mathcal{Q}=\tilde{\mathcal{Z}} \cup\{(\Lambda(\alpha), 0)\}$. We claim that $A$ is a compact subset of $\mathbb{R} \times X$.

Indeed, let $\left\{\left(\lambda_{n}, v_{n}\right)\right\} \subset A$ be an infinitely sequence. Passing toa subsequence, we may suppose that $v_{n}>0$ on $\mathbb{R}^{N}$ forall $n$ and $\lambda_{n} \stackrel{n}{\rightarrow} \lambda \in[\bar{\rho}, \Lambda(\alpha)], v_{n} \rightarrow v$ weakly in $X,\left\|v_{n}\right\| \stackrel{n}{\rightarrow} \tau \geq$ 0 .To prove this claim, it is sufficient to prove $v_{n} \stackrel{n}{\rightarrow} v$ strongly in $X$ with $(\lambda, v) \in A$.If $\tau \geq 0$, then $v_{n} \stackrel{n}{\rightarrow} v$ strongly in $X$. By Lemma 4.1, we must have $(\lambda, v)=(\Lambda(\alpha), 0) \in A$. If $\tau \neq 0$, similar to the proof ofLemma 4.1 , we can deduce that ||$v_{n}-$ $v \| \stackrel{n}{\rightarrow} 0$ and hence $(\lambda, v) \in Z$. The strong maximum implies that $(\lambda, v) \in A$. By a result of Whyburn [11] (see Lemma C.2 in [10]), we can prove that there exists a connected subset $A_{0}$ of $A$ suchthat $A_{0} \cap A_{1} \neq \varnothing$ and $A_{0} \cap A_{2} \neq \varnothing$.It follows that inf $P A_{0}=\bar{\rho}<\rho$. But since $A_{0} \subset \mathcal{C}$, we have infPC $\leq \bar{\rho}<\rho$, a contradiction.

(ii) By contradiction, suppose that there exists a sequence $\left\{\left(\lambda_{n}, v_{n}\right)\right\} \subset \mathcal{C} \backslash\{(\Lambda(\alpha), 0)\}$ such that $\lambda_{n} \stackrel{n}{\rightarrow} \Lambda(\alpha)$ and ||$v_{n}|| \geq \delta>0$ for all $n \in \mathbb{N}$. Bythe proof of part (i) we have ||$v_{n}|| \leq T$. Passing to asubsequence, we may assume that $v_{n} \rightarrow v$ weaklyin $X$. Similar to the proof of Lemma 4.1, we candeduce that $\left\|v_{n}-v\right\| \stackrel{n}{\rightarrow} 0$ and $(\Lambda(\alpha), v) \in Z$. Then by the strong maximum principle wehave $(\Lambda(\alpha), v) \in \tilde{Z}$. But at thebeginning of the proof of part (i) we proved that $\lambda<\Lambda(\alpha)$ for all $(\lambda, v) \in \tilde{Z}$. This is a contradiction.

We have established the global properties of a connected subset of $\tilde{Z} \cup\{(\Lambda(\alpha), 0)\}$ containing $\{(\Lambda(\alpha), 0)\}$. However, in order to maintainconnectedness under inversion, we need to find a connected subset of $\tilde{Z}$ having similar properties.

Set $Z^{+}=\left\{(\lambda, v) \in Z: v>0\right.$ on $\left.\mathbb{R}^{N}\right\}$ and $Z^{-}=\{(\lambda, v) \in$ $Z: v<0$ on $\left.\mathbb{R}^{N}\right\}$.

Corollary 4.1: Let the function $f$ be odd. Then there exist two bounded connectedsubsets $\mathcal{C}_{0}^{+}$and $\mathcal{C}_{0}^{-}$of $Z^{+}$and $Z^{-}$, respectively, satisfying the followingproperties:

(i) $\operatorname{infP} \mathcal{C}_{0}^{ \pm}=\frac{\alpha}{V(\infty)}$ and $(\Lambda(\alpha), 0) \in \overline{\mathcal{C}_{0}^{ \pm}}$.

(ii) $\operatorname{supPC}_{0}^{ \pm}=\Lambda(\alpha)$ and $0<|| v|| \leq T$ for all $(\lambda, v) \in \mathcal{C}_{0}^{ \pm}$, where $T>0$ isgiven by Lemma 3.2.

Proof: The proof is the same as the one of Corollary 5.3 in [10].

\section{Proof of Theorem1.3}

Let $f_{R}$ and $f_{L}$ be the odd functions defined by

$$
f_{R}(s)= \begin{cases}f(x, s), & \text { for } s \geq 0, \\ -f(x,-s), & \text { for } s<0\end{cases}
$$

and $f_{L}(s)=\left\{\begin{array}{l}-f(x,-s), \quad \text { for } s \geq 0 \\ f(x, s), \quad \text { for } s<0 .\end{array}\right.$

By corollary 4.1 there exist two bounded connected subsets $\mathcal{C}_{0}^{+}$and $\mathcal{C}_{0}^{-}$of positive or negativesolutions for problem (1.1) with $f_{R}$ or $f_{L}(s)$ respectively. Setting

$$
\Sigma^{ \pm}=\left\{\left(\lambda, \frac{v}{\mid v \|^{2}}\right):(\lambda, v) \in \mathcal{C}_{0}^{ \pm}\right\}
$$

it follows that $\Sigma^{ \pm}$areconnected sets of $\left(\frac{\alpha}{V(\infty)}, \Lambda(\alpha)\right) \times$ $W^{2, p}\left(\mathbb{R}^{N}\right)$ consisting of, respectively, positive and negative solutions of (1.1) with infP $\sum^{ \pm}=\frac{\alpha}{V(\infty)}, \sup P \sum^{ \pm}=\Lambda(\alpha)$ and ||$u|| \geq \frac{1}{T}$ for all $(\lambda, v) \in \Sigma^{ \pm}$.

Suppose that $\left\{\left(\lambda_{n}, u_{n}\right)\right\} \subset \Sigma^{ \pm} \quad$ with $\lambda_{n} \stackrel{n}{\rightarrow} \lambda>\frac{\alpha}{V(\infty)}$ and $\max _{x \in \mathbb{R}^{N}}\left|u_{n}(x)\right| \stackrel{n}{\rightarrow} \infty$. Then ||$u_{n} \| \stackrel{n}{\rightarrow}+\infty$ by the Sobolev embedding. Hence $\left(\lambda_{n}, v_{n}\right) \in \tilde{Z}$ with $v_{n}=\frac{u_{n}}{\left\|u_{n}\right\|^{2}}$ and $\left\|v_{n}\right\| \stackrel{n}{\rightarrow} 0$. ByLemma 4.1 we have $\lambda=\Lambda(\alpha)$. On the otherhand, if $\left\{\left(\lambda_{n}, u_{n}\right)\right\} \subset \sum^{ \pm}$with $\lambda_{n} \stackrel{n}{\rightarrow} \Lambda(\alpha)$, by setting $v_{n}=\frac{u_{n}}{\left\|u_{n}\right\|^{2}}$ we know that $\left(\lambda_{n}, v_{n}\right) \in \mathcal{C}$. Then by Theorem 4.1 (ii) we have ||$v_{n}|| \stackrel{n}{\rightarrow} 0$. This means that ||$u_{n} \| \stackrel{n}{\rightarrow}+\infty$. We claim that $\max _{x \in \mathbb{R}^{N}}\left|u_{n}\right| \stackrel{n}{\rightarrow}+\infty$. Otherwise, passing to a subsequence, there is $C>0$ such that $\max _{x \in \mathbb{R}^{N}}\left|u_{n}\right| \leq C$ for alln $\in \mathbb{N}$. Since $\left(\lambda_{n}, u_{n}\right)$ is a solution of problem (1.1) we have

$$
L\left(\lambda_{n}\right) u_{n}+k\left(x, u_{n}\right) u_{n}=0
$$

By Lemma 3.3, we know that $u_{n}$ isbounded in $Y$. Therefore, $\left\{(-\Delta+1) u_{n}\right\}$ is bounded in $Y$ by (4.6). Since $-\Delta+1: X \rightarrow Y$ isan isomorphism, this implies that $\left\{u_{n}\right\}$ is bounded in $X$, a contradiction.

[1] T.Bartsch,A.Pankov and Z.Q.Wang, Nonlinear Schödinger equations with steep potential well, Commun. Contemp. Math., 3(2001),549-569.

[2] Y.Ding and K.Tanaka, Multiplicity of positive solutions of a nonlinear Schödinger equation, Manuscripta Math., 112(2003),109-135.

[3] D.G.DE Figueiredo and Y.Ding, Solutions of a non-linear Schrödinger equation, Discrete Contin. Dynam. Systems, 8(2002),563-584.

[4] D.Gilbarg and N.S.Trudinger, it Elliptic Partial Diffential Equations of Second Order, Second edition, Springer-Verlag,Berlin, 1983.

[5] F.A.Van Heerden and Z.Q.Wang, Schrödinger tyoe equations with asymptotically linear nonlinearities, it Differential Integral Equations, 16(2003),257-280.

[6] L.Jeanjean, M.Lucia and C.A.Stuart, Branches of solutions to semilinear ellptic equations on $\mathbb{R}^{N}$, Math. Z., 230 (1999), 79-105.

[7] L.Jeanjean and K.Tanaka, A positive solution for an asymptotically linear elliptic problem on $\mathbb{R}^{N}$ autonomous at infinity, WSAIM Control Optim. Calc. Var., 7(2002), 597-614.

[8] Z. Liu and Z.Q. Wang, Existence of a positive solution of an elliptic equation on $\mathbb{R}^{N}$, Proc. Roy. Soc. Edinburgh, 134 A (2004), 191-200. 
[9] C.A.Stuart, An introduction to elliptic equations on $\mathbb{R}^{N}$, in Nonlinear Functional Analysis and Applications toDifferential Equations, editors A.Ambrosetti, K.C. Chang, I.Ekeland, World Scientific, Singapore, 1998.

[10] C.A.Stuart and Huansong Zhou, Global branch of solutios for nonlinear Schrödinger equations with deepening potential well, Proc.London Math.Soc., 92 (2006) 655-681.

[11] G.T. Whyburn, Topological Analysis, Princeton University Press, Preceton 1958. 